\begin{tabular}{|c|l|}
\hline Title & $\begin{array}{l}\text { A new method to improve validity range of Lie canonical perturbation theory : with a central focus on a concept of non- } \\
\text { blow-up region }\end{array}$ \\
\hline Author(s) & Teramoto, Hiroshi; Toda, Mikito; Komatsuzaki, Tamiki \\
\hline Citation & $\begin{array}{l}\text { Theoretical chemistry accounts, 133(11), 1571(1)-1571(15) } \\
\text { https:/doi.org/_0.1007/300214_014_1571-9 }\end{array}$ \\
\hline Issue Date & 201411 \\
\hline Doc URL & http://hdl.handle.net/2115/59809 \\
\hline Rights & The final publication is available at link.springer.com \\
\hline Type & article (author version) \\
\hline Note & Published online 13 September 2014 \\
\hline
\end{tabular}

Instructions for use 


\title{
A New Method to Improve Validity Range of Lie Canonical Perturbation Theory With a Central Focus on a Concept of Non Blow-Up Region
}

\author{
Hiroshi Teramoto - Mikito Toda • \\ Tamiki Komatsuzaki
}

Received: date / Accepted: date

\begin{abstract}
Validity ranges of Lie Canonical Perturbation Theory (LCPT) are investigated in terms of non blow-up regions. We investigate how the validity ranges depend on the perturbation order in two systems, one of which is a simple Hamiltonian system with one degree of freedom and the other is a $\mathrm{HCN}$ molecule. Our analysis of the former system indicates that non blow-up regions become reduced in size as the perturbation order increases. In case of LCPT by Dragt and Finn and that by Deprit, the non blow-up regions enclose the region inside the separatrix of the Hamiltonian but it may not be the case for LCPT by Hori. We also analyze how well the actions constructed by these LCPTs approximate the true action of the Hamiltonian in the non blow-up regions and find that the conventional truncated LCPT does not work over the whole region inside the separatrix whereas LCPT by Dragt and Finn without truncation does. Our analysis of the latter system indicates that non blow-up regions do not necessarily cover the whole regions inside the HCN well.

We propose a new perturbation method to improve non blow-up regions and validity ranges inside them. Our method is free from blowing up and

\footnotetext{
Hiroshi Teramoto

Molecule \& Life Nonlinear Sciences Laboratory, Research Institute for Electronic Science, Hokkaido University, Kita 20 Nishi 10, Kita-ku, Sapporo 001-0020, Japan

Tel.\&Fax: +81-11-706-9436

E-mail: teramoto@es.hokudai.ac.jp

Mikito Toda

Nonequilibrium Dynamics Laboratory, Research group of Physics, Division of Natural Science, Nara Women's University, Nara, 630-8506, Japan

Tel.\&Fax: +81-742-20-3383

E-mail: toda@ki-rin.phys.nara-wu.ac.jp

Tamiki Komatsuzaki

Molecule \& Life Nonlinear Sciences Laboratory, Research Institute for Electronic Science, Hokkaido University, Kita 20 Nishi 10, Kita-ku, Sapporo 001-0020, Japan

Tel.\&Fax: +81-11-706-9434

E-mail: tamiki@es.hokudai.ac.jp
} 
retains the same normal form as the conventional LCPT. We demonstrate our method in the two systems and show that the actions constructed by our method have larger validity ranges than those by the conventional and our previous methods proposed in $[1,2]$.

Keywords Lie Canonical Perturbation Theory · Non Blow-Up Region • HCN Isomerization

\section{Introduction}

Canonical transformations are coordinate transformations in the phase space of Hamiltonian systems that preserve symplectic two forms, i.e., preserving the form of Hamiltonian equations of motions. Canonical perturbation theory (CPT) is one of the fundamental theories of solving nonlinear dynamical problems that is carried by perturbation from integrable systems through some canonical transformation. CPT has often been applied for seeking for integrals of motions, adiabatic invariants and a better and simpler description of the systems [3]. The traditional canonical transformation is by mixed-variable generating function composed of old and new canonical variables. The most traditional Poincaré-Von Zeipel CPT [3] based on the mixed-variable generating function approach, however, imposes a major impediment to implementing higher order perturbations. Among CPTs, Lie canonical perturbation theory (LCPT) originally developed by Hori[4,5] and Deprit[6], later by Dragt and Finn[7], is very powerful in that canonical transformation is carried by a series of operations of Poisson brackets avoiding cumbersome generating function of mixed variables where complete inversion from the old to the new canonical variables is rather straightforward. Their mutual relation and their computational efficiency have also been investigated [8-11]. These different formats result in the same normal form Hamiltonian but these formats can result in different normal form transformations. The convergence or divergence of the normal form and normal form transformation of order infinity has been investigated in the previous studies [12-24] under various conditions. Under some of these conditions, the normal form and normal form transformation converges globally, but, since generic Hamiltonians are non-integrable [25], in most cases, there is no hope to seek for (nontrivial) global integral of motions without any symmetries. Under such circumstance, what one can do best is to look into a better and simpler local description of the system in question. LCPT has been applied to seeking for such local descriptions in a perturbative manner from integrable solutions, and shown to be versatile in various types of Hamiltonian in the research fields such as celestial mechanics[26, 27], atomic physics[28,29], cluster physics[30-36]. For example, in the context of chemical reaction dynamics, LCPT has been applied to seeking (locally-)no-return transition state and the associated reaction coordinate buried in the phase space for many-degrees of freedom Hamiltonian systems such as intramolecular proton transfer in malonaldehyde [37,38], argon cluster isomerization [3036], $\mathrm{O}\left({ }^{1} D\right)+\mathrm{N}_{2} \mathrm{O} \rightarrow \mathrm{NO}+\mathrm{NO}[39]$, a hydrogen atom in crossed electric and 
magnetic fields [29,40], HCN isomerization [41,42,1,2], and so forth. LCPT was generalized to dissipative systems such as multidimensional (generalized) Langevin formulation to describe reactions under thermal fluctuation, in which no-return transition state can be obtained by incorporating nonlinearity of the system and interactions with heat bath [43-50]. The pioneering studies on semiclassical analog of LCPT was also carried out in late 1980s for multidimensional resonant, nonresonant, and nearly resonant systems[51-53]: They presented a method for deriving corrections in powers of Planck's constant by the reflection of the underlying (near) divergence properties of classical chaos, which was found to be effective even at low order corrections in improving the accuracy of the energy eigenvalues. Recently, their semiclassical studies were extended to the analyses of reaction dynamics over a rank-one saddle under a time-dependent external field (optimally controlled laser pulse), and it was found that optimally controlled laser pulse corresponds to modulating the boundary of the reaction in the phase space so as to catch the system excited in the reactant well and then to release it into the product [54]. This method provides a new protocol to design the laser field facilitated by the classical phase space picture [55].

However, in most cases, the convergence radii of these LCPTs are limited even for finite order of perturbations [56], and the convergence radii shrink to zero as the perturbation order increases. In a context of chemical reaction dynamics, molecules exhibit larger amplitude motions as their energies increase. For these molecules to surmount the reaction barriers, they must have large enough energies. Therefore, to describe and understand the chemical reaction dynamics, it is vital to develop a perturbation method that is valid not only in the very vicinity of their equilibrium structures but also in regions far from them. In a broader context, if we succeed in obtaining a better estimation for approximate invariants of motion, we would be able to analyze dynamics not only for near-integrable systems but also for systems with mixed phase space, i.e., those systems which exhibit both chaotic and regular behavior.

In the study of systems with mixed phase space, one of the crucial problems is to find boundaries between chaotic and regular behavior. For systems of more than two degrees of freedom, it is well known that the KAM tori do not divide the equi-energy surface into two separate regions. In fact, Arnold showed, for a specific model Hamiltonian, that trajectories detour around KAM tori, thereby leading to the motion along the resonances [57]. Such motions are now called the Arnold diffusion [58-60]. Moreover, it is known that the resonances constitute a network called the Arnold web [3,61,62,28,63-65], where the motion across the resonances gives rise to faster diffusion especially around resonance junctions $[66,67]$. Thus, in the analysis of the dynamics on the Arnold web, it is crucial to find those regions which trap trajectories for a finite but longer duration [68], since distribution of resonances plays a key role for statistical features of the reaction dynamics $[69,70]$. Then, better approximate invariants would offer a clue to find how chaotic and regular regions are distributed in the phase space. 
Another important issue in systems of mixed phase space is the transport in the phase space [71-73], i.e., to understand how different regions of the phase space are dynamically connected. In chemistry, reaction processes are nothing but the transport from a potential well to another one via a saddle region. Thus, we face the problem of what kind of phase space structures connect dynamics in a well to that in another one [74]. In such studies, we need to construct better action variables, if any, in different regions of the phase space so that validity ranges of different sets of variables overlap with each other. Then, we could investigate the connection based on the transformation between different sets of the approximate invariants corresponding to different regions of the phase space.

Teramoto et al. $[1,2]$ proposed a method that makes LCPT valid in wider regions than those in the previous method and demonstrated it in a highlyexcited HCN molecule. The crux is to calculate canonical transformation in each order of LCPT without any truncation errors. However, validity ranges of their method are also limited by non blow-up regions. Validity range of a LCPT is a subset of phase space where the resultant normal form is valid. For example, if the normal form is to construct slowly varying actions, center manifolds or stable and unstable manifolds, the validity range of the LCPT is a region where the resultant normal form describes these objects within a given accuracy needed to describe systems. Non blow-up region of LCPT is a subset of initial conditions in the phase space where the results of the perturbation are finite. Non blow-up region limits the validity ranges of LCPT because the results should be at least finite to validate them. To improve their method further, it is important to understand these concepts. Sec. 2 is devoted to an illustration of these concepts in a simple one-degree-of-freedom Hamiltonian system and elucidations of these concepts along with their numerical demonstrations. In Sec. 3, we propose a new perturbation method to avoid blowing up while retaining the normal forms and demonstrate it in Sec. 4. Sec. 5 is devoted to conclusions and discussions.

\section{Non blow-up regions of Lie canonical perturbation theory, LCPT}

2.1 An illustration of a validity range of LCPT for a one-dimensional Hamiltonian system

To illustrate non blow-up regions of LCPT, let us investigate a simple Hamiltonian system. Let $(q, p)$ be a coordinate and its conjugate momentum with a Hamiltonian represented by

$$
H(q, p)=\frac{1}{2}\left(p^{2}+q^{2}\right)+\left(2 p^{2} q-q^{3}\right) .
$$

LCPT seeks for a canonical transformation $(q, p) \mapsto(Q, P)$ so that the Hamiltonian (Eq. (1)) in terms of the new coordinate $(Q, P)$ becomes simple in a 
certain sense. There exist several conventions for the simplicities [11] and normal forms that attain them ${ }^{1}$. In this specific example, the leading order of normal form would be like

$$
\bar{H}(Q, P)=\frac{1}{2}\left(P^{2}+Q^{2}\right)+O(4),
$$

$(O(4)$ means a collection of terms of order quartic and those of higher than quartic with respect to $P$ and $Q$.) such that it has the same quadratic terms but does not have terms of order cubic. To obtain the normal form, LCPT seeks for a canonical transformation generated by a generating function $F(q, p)$, i.e.,

$$
\begin{aligned}
& Q(q, p)=e^{-\{F(q, p) \cdot \cdot\}} q, \\
& P(q, p)=e^{-\{F(q, p) \cdot \cdot\}} p,
\end{aligned}
$$

where $\{\cdot, \cdot\}$ is Poisson bracket defined as

$$
\{A(q, p), B(q, p)\}=\frac{\partial A(q, p)}{\partial q} \frac{\partial B(q, p)}{\partial p}-\frac{\partial A(q, p)}{\partial p} \frac{\partial B(q, p)}{\partial q} .
$$

A benefit for Lie canonical transformation is that the inverse transformation $(Q, P) \rightarrow(q, p)$ can be easily written as

$$
\begin{aligned}
& q(Q, P)=e^{\{F(Q, P), \cdot\}} Q, \\
& p(Q, P)=e^{\{F(Q, P), \cdot\}} P .
\end{aligned}
$$

This can be evaluated by using the following relation,

$$
\begin{aligned}
\left\{A^{\prime}(Q, P), B^{\prime}(Q, P)\right\} & =\frac{\partial A^{\prime}(Q(q, p), P(q, p))}{\partial q} \frac{\partial B^{\prime}(Q(q, p), P(q, p))}{\partial p} \\
& -\frac{\partial A^{\prime}(Q(q, p), P(q, p))}{\partial p} \frac{\partial B^{\prime}(Q(q, p), P(q, p))}{\partial q}, \quad(8) \\
& =\frac{\partial A^{\prime}(Q, P)}{\partial Q} \frac{\partial B^{\prime}(Q, P)}{\partial P}-\frac{\partial A^{\prime}(Q, P)}{\partial P} \frac{\partial B^{\prime}(Q, P)}{\partial Q}(9)
\end{aligned}
$$

which holds for arbitrary differentiable functions $A^{\prime}(Q, P)$ and $B^{\prime}(Q, P)$ if the transformation $(q, p) \mapsto(Q, P)$ is a canonical transformation [75]. By using Eq. (10), the leading order expression of Eqs. (6) and (7) can be written as

$$
\begin{aligned}
& q(Q, P)=Q-\frac{\partial F(Q, P)}{\partial P}+\cdots, \\
& p(Q, P)=P+\frac{\partial F(Q, P)}{\partial Q}+\cdots .
\end{aligned}
$$

\footnotetext{
1 In a context of chemistry, people are often interested in extracting slowly varying action variables, i.e., adiabatic invariants. It is because these variables determine the slowest time scale of intra-molecular vibrational relaxation and chemical reaction triggered by that. For this purpose, the desired normal form would be the one that maximally decouples these action variables from those of the other degrees of freedom.
} 
By plugging the leading order expression in Eq. (1), we get

$$
\begin{aligned}
\bar{H}(Q, P) & =H(p, q) \\
& =\frac{1}{2}\left(Q^{2}+P^{2}\right)+\left(2 P^{2} Q-Q^{3}\right)-Q \frac{\partial F(Q, P)}{\partial P}+P \frac{\partial F(Q, P)}{\partial Q}+\cdots .
\end{aligned}
$$

To eliminate the cubic term of Eq. (14), the generating function $F(Q, P)$ should satisfy

$$
\left(2 P^{2} Q-Q^{3}\right)-Q \frac{\partial F(Q, P)}{\partial P}+P \frac{\partial F(Q, P)}{\partial Q}=0 .
$$

Eq. (15) can have multiple solutions but, in this specific case, the conventional semi-simple normal form requires

1. $F(Q, P)$ is of order cubic.

2. $F(Q, P) \in \operatorname{Im}\left(-Q \frac{\partial}{\partial P}+P \frac{\partial}{\partial Q}\right)=\operatorname{Im}\left\{H_{2}(Q, P), \cdot\right\}$, where $H_{2}(Q, P)=$ $\frac{1}{2}\left(P^{2}+Q^{2}\right)$ is the quadratic term of Eq. (2) and $\operatorname{Im} A$ is the image of the operator $A$, i.e., $\operatorname{Im} A=\{f \mid \exists g, f=A g\}$.

By these requirements, the solution of Eq. (15) becomes unique and $F(Q, P)=$ $-P Q^{2}$. With this choice, the $\cdots$ terms in Eq. (14) becomes of order quartic and the canonical transformation generated by the generating function $F(Q, P)$ is actually what we sought for. In this case, we can exactly calculate the canonical transformation (Eqs. (3) and (4)) and we get

$$
\begin{aligned}
& Q(q, p)=\frac{q}{1+q}, \\
& P(q, p)=p(q+1)^{2},
\end{aligned}
$$

by using the fact that the canonical transformations can be calculated by integrating the following differential equations up to $\epsilon=1$,

$$
\begin{aligned}
\frac{d q(\epsilon)}{d \epsilon} & =\frac{\partial F(q(\epsilon), p(\epsilon))}{\partial p}, \\
\frac{d p(\epsilon)}{d \epsilon} & =-\frac{\partial F(q(\epsilon), p(\epsilon))}{\partial q},
\end{aligned}
$$

starting from the initial condition $(q(0), p(0))=(q, p)$ at $\epsilon=0$. Then, $Q(q, p)$ and $P(q, p)$ can be obtained as $(Q(q, p), P(q, p))=(q(1), p(1))$.

Note that the canonical transformation has a set of singular points at $q=$ -1 . Therefore, the maximally connected component containing the origin and where the canonical transformation is well-defined is $\operatorname{Dom}_{F}=\{(q, p) \mid-1<q\}$. We call $\operatorname{Dom}_{F}$ the non blow-up region of the canonical transformation generated by $F$. As long as one uses the formal power series of the canonical transformation, its domain of convergence cannot go beyond this region and thus this region limits the validity range of Lie canonical perturbation theory. 
To illustrate this, let us consider the power series expansion of the canonical transformation (Eqs. (16) and (17)),

$$
\begin{aligned}
& Q(q, p)=\sum_{l=0}^{\infty}(-1)^{l} q^{l+1}, \\
& P(q, p)=p q^{2}+2 p q+p .
\end{aligned}
$$

The region where this expansion converges is $\{(q, p) \mid-1<q<1\}$, which is strictly smaller than $\operatorname{Dom}_{F}$. Roughly speaking, the convergence radius of a canonical transformation is determined by the shortest distance between the expansion origin and the singularity of the canonical transformation and the expansion converges only within an isotropic circle with the radius ${ }^{2}$. However, if its non blow-up region extends anisotropically in the phase space, like the current example, the non blow-up region can be much larger than the region where the expansion converges.

\subsection{Non-blow up regions of LCPT for $n$-dimensional Hamiltonian systems}

Let $\mathbf{q}=\left(q_{1}, \cdots, q_{n}\right)$ be coordinates in an $n$-dimensional Hamiltonian system and $\mathbf{p}=\left(p_{1}, \cdots, p_{n}\right)$ be their conjugate momenta with a Hamiltonian of the system $H(\mathbf{q}, \mathbf{p})$, which is analytic in a neighborhood of the origin $(\mathbf{q}, \mathbf{p})=\mathbf{0}$. In addition, let the Hamiltonian have a stationary point at the origin $(\mathbf{q}, \mathbf{p})=\mathbf{0}$, i.e., $\left.\left(\frac{\partial H(\mathbf{q}, \mathbf{p})}{\partial \mathbf{p}},-\frac{\partial H(\mathbf{q}, \mathbf{p})}{\partial \mathbf{q}}\right)\right|_{(\mathbf{q}, \mathbf{p})=\mathbf{0}}=\mathbf{0}$. Without loss of generality, the value of the Hamiltonian at the origin can be set to zero, i.e., $H(\mathbf{0}, \mathbf{0})=0$. Under these settings, in a neighborhood of the origin, the Hamiltonian can be written as

$$
H(\mathbf{q}, \mathbf{p})=\sum_{k=2}^{\infty} H_{k}(\mathbf{q}, \mathbf{p})
$$

where $H_{k}(\mathbf{q}, \mathbf{p})$ is a homogeneous polynomial of order $k$ with respect to $(\mathbf{q}, \mathbf{p})$. Depending on the form of $H_{2}(\mathbf{q}, \mathbf{p})$, several types of normal forms have been proposed, such as, semi-simple normal form, inner product normal form [11]. There also exist several types of the normalization procedures to realize the normal forms [11]. Here, we use the normalization procedure due to Dragt and Finn [77], which is classified as format 2a in $[11]^{3}$. However, our method works for other procedures classified into format 2 in [11]. The procedure of Dragt and Finn aims at normalizing the Hamiltonian (Eq. (22)) by the following

\footnotetext{
2 Note that, if the generating function $F$ is real analytic, $Q(q, p)$ and $P(q, p)$ are also real analytic in $\operatorname{Dom}_{F}[76]$.

3 In this book, the existing types of perturbations are classified to the five formats, format $1 \mathrm{a}, 1 \mathrm{~b}, 2 \mathrm{a}, 2 \mathrm{~b}$, and $2 \mathrm{c}$, depending on whether they use generating function or not, iterative or recursive. LCPTs by Dragt and Finn, Hori, and Deprit are classified into format 2a, 2b, and $2 \mathrm{c}$, respectively. For details of the classification, see [11] in Sec. 3.2.
} 
consecutive Lie canonical transformations

$$
\begin{aligned}
& \mathbf{Q}^{(m)}(\mathbf{q}, \mathbf{p})=e^{-\left\{F_{m}, \cdot\right\}} e^{-\left\{F_{m-1}, \cdot\right\}} \cdots e^{-\left\{F_{3}, \cdot\right\}} \mathbf{q} \\
& \mathbf{P}^{(m)}(\mathbf{q}, \mathbf{p})=e^{-\left\{F_{m}, \cdot\right\}} e^{-\left\{F_{m-1}, \cdot\right\}} \cdots e^{-\left\{F_{3}, \cdot\right\}} \mathbf{p}
\end{aligned}
$$

generated by the generating functions $F_{m}, F_{m-1}, \cdots, F_{3}$ where $F_{k}(3 \leq k \leq m)$ is a homogeneous polynomial of order $k$ with respect to $(\mathbf{q}, \mathbf{p})$. The non blowup region $U_{m}$ of the LCPT is

$$
\begin{aligned}
U_{m} & =\operatorname{Dom}_{F_{3}} \cap \bigcap_{k=4}^{m} e^{\left\{F_{3}, \cdot\right\}} \ldots e^{\left\{F_{k-1}, \cdot\right\}} \operatorname{Dom}_{F_{k}}, \\
& =\operatorname{Dom}_{F_{3}} \cap e^{\left\{F_{3}, \cdot\right\}} \operatorname{Dom}_{F_{4}} \cap \cdots \cap e^{\left\{F_{3}, \cdot\right\}} \cdots e^{\left\{F_{m-1}, \cdot\right\}} \operatorname{Dom}_{F_{m}},
\end{aligned}
$$

that is, an intersection among $\operatorname{Dom}_{F_{3}}$ and $e^{\left\{F_{3}, \cdot\right\}} \ldots e^{\left\{F_{k-1}, \cdot\right\}} \operatorname{Dom}_{F_{k}}(k=4, \cdots, m)$, which is the non blow-up region Dom $F_{k}$ pulled back to the space spanned by the original phase space variables, $\mathbf{p}$ and $\mathbf{q}$.

In general, we have $U_{m_{1}} \subseteq U_{m_{2}}$ for $m_{1} \geq m_{2}$ and thus the non blow-up region shrinks as the perturbation order $m(m \geq 3)$ increases. The question of how the non blow-up region shrinks depends on specific forms of the generating functions but, in general, if $k(k \geq 3)$ is odd and if $\mathbf{0}$ is an isolated critical point of $F_{k}(\mathbf{q}, \mathbf{p})^{4}$, the differential equation induced by $F_{k}(\mathbf{q}, \mathbf{p})$,

$$
\begin{aligned}
& \frac{d \mathbf{q}}{d \epsilon}=\frac{\partial F_{k}(\mathbf{q}, \mathbf{p})}{\partial \mathbf{p}}, \\
& \frac{d \mathbf{p}}{d \epsilon}=-\frac{\partial F_{k}(\mathbf{q}, \mathbf{p})}{\partial \mathbf{q}},
\end{aligned}
$$

is unbounded, i.e., there is at least one unbounded solution $[78]^{5}$. If the unbounded solution blows up in a finite time, it can be shown that non blow-up region of the canonical transformation generated by $F_{k}$ is not equal to the whole phase space. The reason is the following. Let $k$ be an odd integer that is larger than 2 and $(\mathbf{q}(\epsilon), \mathbf{p}(\epsilon))$ be one of the solutions of the differential equation that blows up at $\epsilon^{*}$. Then,

$$
\left(\mathbf{q}^{\prime}(\epsilon), \mathbf{p}^{\prime}(\epsilon)\right)=\left(\epsilon^{*}\right)^{\frac{1}{k-2}}\left(\mathbf{q}\left(\epsilon^{*} \epsilon\right), \mathbf{p}\left(\epsilon^{*} \epsilon\right)\right)
$$

is also the solution of the differential equation that blows up at $\epsilon=1$.

\footnotetext{
$4 \mathbf{0}$ is an isolated critical point of $F_{k}(\mathbf{q}, \mathbf{p})$ if $F_{k}(\mathbf{q}, \mathbf{p})$ has a critical (stationary) point at $\mathbf{0}$ and there exists an open neighborhood of $\mathbf{0}$ within which there is no critical point other than 0.

5 See COROLLARY in [78] in p. 1921. Note that $k$ in this manuscript corresponds to $k+1$ in their notation.
} 
2.3 A Demonstration of how the non blow-up region $U_{m}$ depends on the perturbation order $m$

In this section, we provide two examples of how the non blow-up region $U_{m}$ shrinks as the perturbation order $m$ increases. First, we evaluate non blow-up regions of LCPT by Dragt and Finn in a Hamiltonian (Eq. (1)) in Sec. 2.3.1 and compare them with those of LCPTs by Hori and by Deprit, and, then, evaluate non blow-up regions in a HCN molecule in Sec. 2.3.2. In both the examples, we use a blow-up technique to integrate the differential equation in Eqs. (27) and (28) shown in Sec. 7.1 in Appendix.

\subsubsection{Non blow-up regions in a Hamiltonian (Eq. (1))}

In this section, we investigate how the non blow-up region $U_{m}$ depends on the perturbation order $m$. To investigate their relation to the phase space topology of the Hamiltonian (Eq. (1)), we plot contour lines of the Hamiltonian in Fig. 1 (a) in the energy range $[-0.1,0.4]$. This Hamiltonian has four fixed points, one of which is elliptic and the other three are hyperbolic. The elliptic fixed point is located at the origin $(q, p)=\mathbf{0}$ and the other three are located at $\left(\frac{1}{3}, 0\right)$, $\left(-\frac{1}{4}, \sqrt{\frac{7}{32}}\right)$ and $\left(-\frac{1}{4},-\sqrt{\frac{7}{32}}\right)$, respectively. The one located at $\left(\frac{1}{3}, 0\right)$ has an energy $\frac{1}{54}$ that is smaller than that of the other two hyperbolic fixed points and thus the closest separatrix from the origin is made up of the stable and unstable manifolds of the hyperbolic fixed point $\left(\frac{1}{3}, 0\right)$. In Fig. 1 (b), we plot non blow-up regions $U_{m}(m=5,10,15,20)$ of LCPT by Dragt and Finn in the Hamiltonian (Eq. (1)) along with the separatrix of the Hamiltonian. From this figure, $U_{m}$ shrinks as $m$ increases and $U_{m}$ converges into the region inside the separatrix. To see if the similar behavior can be seen in other types of perturbation theory, we compare non blow-up regions of LCPT by Hori, and that by Deprit which are classified as format $2 \mathrm{~b}$ and $2 \mathrm{c}$ in [11], respectively. The former one seeks for the canonical perturbation of the form

$$
\begin{aligned}
& \tilde{Q}^{(m)}(q, p)=e^{-\sum_{k=3}^{m}\left\{\tilde{F}_{k}, \cdot\right\}} q, \\
& \tilde{P}^{(m)}(q, p)=e^{-\sum_{k=3}^{m}\left\{\tilde{F}_{k}, \cdot\right\}} p,
\end{aligned}
$$

where $\tilde{F}_{k}(q, p)(k=3, \cdots, m)$ is a homogeneous polynomial of order $k$ with respect to $q$ and $p$. The generating function $\tilde{F}_{k}(q, p)(k=3, \cdots, m)$ is determined by the conventional manner (see [11].). $\tilde{Q}^{(m)}(q, p)$ and $\tilde{P}^{(m)}(q, p)$ can be obtained by integrating the differential equations

$$
\begin{aligned}
& \frac{d q(\epsilon)}{d \epsilon}=\frac{\partial \sum_{k=3}^{m} \tilde{F}_{k}(q(\epsilon), p(\epsilon))}{\partial p}, \\
& \frac{d p(\epsilon)}{d \epsilon}=-\frac{\partial \sum_{k=3}^{m} \tilde{F}_{k}(q(\epsilon), p(\epsilon))}{\partial q} .
\end{aligned}
$$

up to $\epsilon=1$ starting from $(q(0), p(0))=(q, p)$ at $\epsilon=0$ and by putting

$$
\left(\tilde{Q}^{(m)}(q, p), \tilde{P}^{(m)}(q, p)\right)=(q(1), p(1)) .
$$


(a)

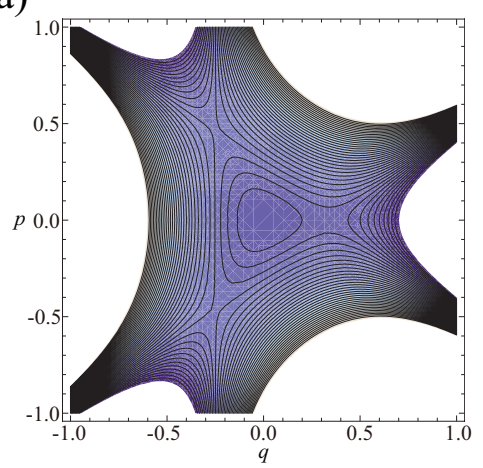

(c)

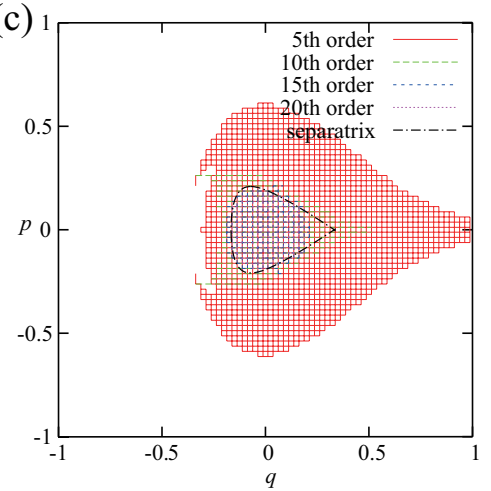

(b)

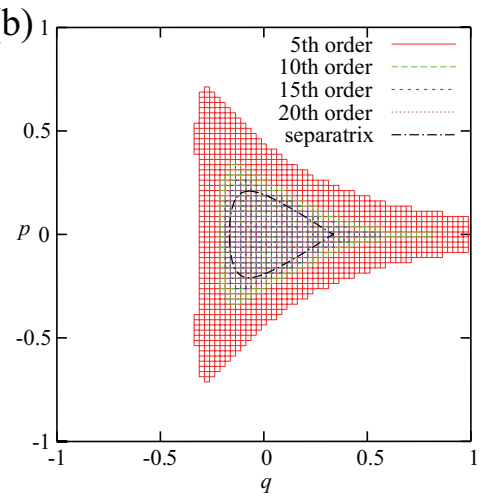

(d)

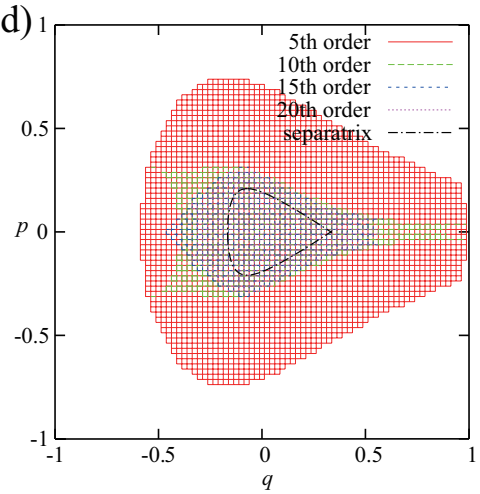

Fig. 1 (a) Contour lines of the Hamiltonian (Eq. (1)), (b,c,d) Non blow-up regions of LCPT by (b) Dragt and Finn $U_{m}(m=5,10,15,20)$, (c) Hori $\tilde{U}_{m}(m=5,10,15,20)$, and, (d) Deprit $\hat{U}_{m}(m=5,10,15,20)$, along with the separatrix of the Hamiltonian (Eq. (1)).

The latter one seeks for a canonical transformation generated by the generating function $W^{(m)}(\epsilon, q, p)=\sum_{k=3}^{m} \frac{\epsilon^{k-3}}{(k-3) !} \hat{F}_{k}(q, p)$, where $\hat{F}_{k}(q, p)$ is a homogeneous polynomial of order $k$. In this case, the new variables $\left(\hat{Q}^{(m)}(q, p), \hat{P}^{(m)}(q, p)\right)$ can be obtained by integrating the differential equation,

$$
\begin{aligned}
& \frac{d q(\epsilon)}{d \epsilon}=\frac{\partial W^{(m)}(\epsilon, q(\epsilon), p(\epsilon))}{\partial p}, \\
& \frac{d p(\epsilon)}{d \epsilon}=-\frac{\partial W^{(m)}(\epsilon, q(\epsilon), p(\epsilon))}{\partial q},
\end{aligned}
$$

until $\epsilon=1$, starting from the initial condition $(q(0), p(0))=(q, p)$ at $\epsilon=0$. Then, $\left(\hat{Q}^{(m)}(q, p), \hat{P}^{(m)}(q, p)\right)$ can be obtained as $\left(\hat{Q}^{(m)}(q, p), \hat{P}^{(m)}(q, p)\right)=$ $(q(1), p(1))$. In these cases, we also define non blow-up regions of the LCPT of order $m, \tilde{U}_{m}$ (Hori) and $\hat{U}_{m}$ (Deprit) as the set of the initial conditions where the solutions of the canonical transformations generated by these generating functions are bounded. In Fig. 1 (c) and (d), we plot $\tilde{U}_{m}$ and $\hat{U}_{m}$ for 
$m=5,10,15$ and 20 . For Hori's LCPT, the non blow-up regions $\tilde{U}_{15}$ and $\tilde{U}_{20}$ do not cover the whole region inside the separatrix whereas those of Deprit's LCPT cover relatively wide regions in the phase space. In this specific example, LCPT by Dragt and Finn and that by Deprit have wider non blow-up regions up to the perturbation order 20th than that by Hori. More systematic study is needed to determine the best format of all the possible formats of LCPT (some of them is listed in [11]) that leads to the widest non blow-up region among them.

To investigate validity ranges of the LCPTs, we compare the action variables constructed by using the LCPTs with the true action inside the separatrix. Here, the true action is defined as

$$
I=\frac{1}{2 \pi} \int_{\{(q, p) \mid H(q, p) \leq E, \text { inside the separatrix }\}} d q d p,
$$

[75] while the actions constructed by LCPTs are denoted as $I^{(20)}=\frac{1}{2}\left(\left(p^{(20)}\right)^{2}+\left(q^{(20)}\right)^{2}\right)$ (Dragt and Finn), $\tilde{I}^{(20)}=\frac{1}{2}\left(\left(\tilde{p}^{(20)}\right)^{2}+\left(\tilde{q}^{(20)}\right)^{2}\right)($ Hori $)$, and $\hat{I}^{(20)}=\frac{1}{2}\left(\left(\hat{p}^{(20)}\right)^{2}+\left(\hat{q}^{(20)}\right)^{2}\right)$ (Deprit) with $I_{\text {trunc }}^{(20)}=\frac{1}{2}\left(\left(p_{\text {trunc }}^{(20)}\right)^{2}+\left(q_{\text {trunc }}^{(20)}\right)^{2}\right)$ (Dragt and Finn, truncated), respectively, where $p_{\text {trunc }}^{(20)}$ and $q_{\text {trunc }}^{(20)}$ are constructed as follows. First, expand the canonical transformation Eqs. (23) and (24) with respect to $\mathbf{q}$ and p, and then, truncated it at the order 21-st, which is the conventional prescription used in [29]. These actions are close with each other within $O\left(E^{\frac{21}{2}}\right)$. It is because the Hamiltonian (1) can be written as $H(q, p)=H_{\text {int }}\left(I^{(20)}\right)+O(21)$ (this symbol $O$ is the same as that defined in Eq. (2)) in terms of these actions, and, thus, the following equation

$$
\begin{aligned}
I & =\frac{1}{2 \pi} \int_{\left\{(q, p) \mid H_{\mathrm{int}}\left(I^{(20)}\right) \leq E, \text { inside the separatrix }\right\}} d q d p+O\left(E^{\frac{20+1}{2}}\right) \\
& =\frac{1}{2 \pi} \int_{\left\{(q, p) \mid H_{\mathrm{int}}\left(I^{(20)}\right) \leq E, \text { inside the separatrix }\right\}} d I^{(20)} d \Theta^{(20)}+O\left(E^{\frac{21}{2}}\right), \\
& =\int_{\left\{I^{\prime} \mid H_{\mathrm{int}}\left(I^{\prime}\right) \leq E\right\}} d I^{(20)}+O\left(E^{\frac{21}{2}}\right) \\
& =I^{(20)}+O\left(E^{\frac{21}{2}}\right)
\end{aligned}
$$

holds. To derive the last equality, we use the fact that $H_{\text {int }}\left(I^{\prime}\right)$ is monotonically increasing with respect to $I^{\prime}$. Therefore, the difference between the action $I^{(20)}$ and the true action is $O\left(E^{\frac{21}{2}}\right)$. The same is true for the other actions. Note that, at energies above that of the separatrix, contour lines of the Hamiltonian do not enclose finite regions and, thus, the true action is defined only inside the separatrix. However, the actions constructed by using the LCPTs are well-defined inside their non blow-up regions and we call them actions in what follows. In Fig. 2 (a), (b), (c), and (d), we show that their relative errors from 

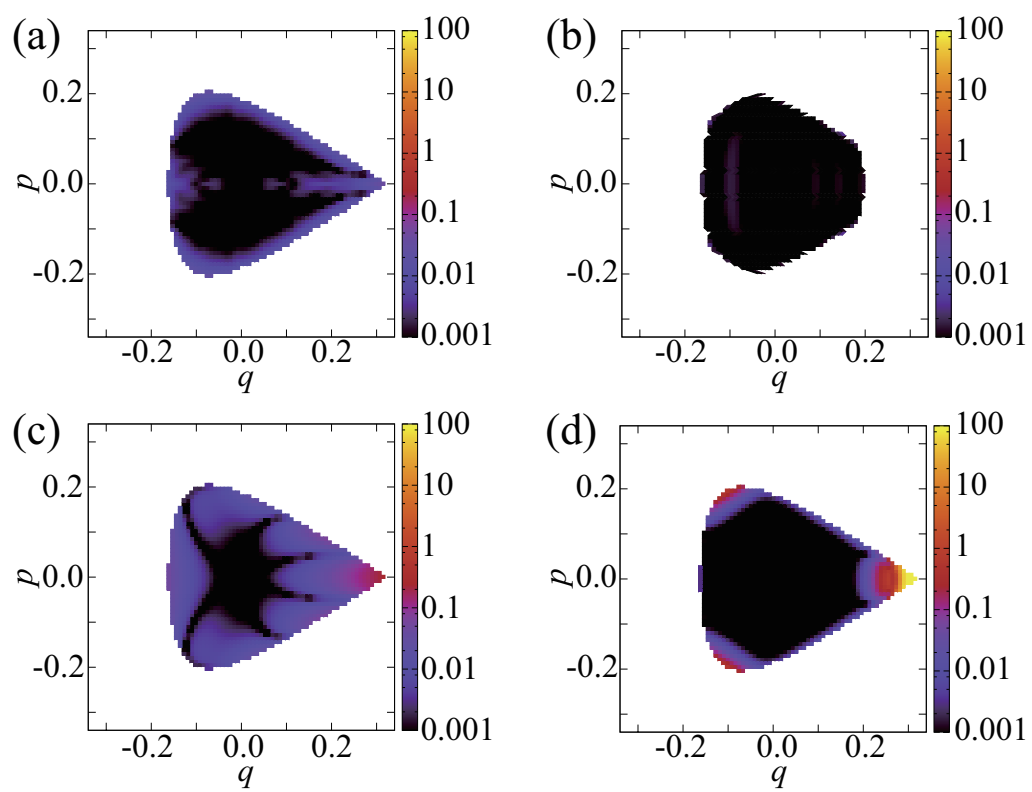

Fig. 2 (a-d) The relative error between the true action $I$ and (a) $I^{(20)}$ (Dragt and Finn), (b) $\tilde{I}^{(20)}$ (Hori), (c) $\hat{I}^{(20)}$ (Deprit), and (d) $I_{\text {trunc }}^{(20)}$ (Dragt and Finn, truncated), plotted inside the separatrix.

the true action $I$, defined as (a) $\frac{\left|I^{(20)}-I\right|}{I}$ (Dragt and Finn), (b) $\frac{\left|\tilde{I}^{(20)}-I\right|}{I}$ (Hori), (c) $\frac{\left|\hat{I}^{(20)}-I\right|}{I}$ (Deprit), and (d) $\frac{\left|I_{\text {trunc }}^{(20)}-I\right|}{I}$ (Dragt and Finn, truncated), respectively. This comparison shows that the truncated one, $I_{\text {trunc }}^{(20)}$ cannot describe the true action properly at the region close to the separatrix (the relative error exceeds 100.) whereas $I^{(20)}$, describes the action inside the separatrix within one percent error. This tendency does not change even if the perturbation order is increased further. In addition, $\hat{I}^{(20)}$ (Deprit) has larger errors than $I^{(20)}$ (Dragt and Finn), whereas $\tilde{I}^{(20)}$ (Hori) has errors comparable to those $I^{(20)}$ (Dragt and Finn) has inside of the non blow-up region $\tilde{U}_{20}$. More systematic study is needed to be done but, in this specific example, the LCPT by Dragt and Finn leads to the best result among all, regarding the width of the non blow-up region and the accuracy inside of it. Therefore, we use the LCPT by Dragt and Finn in what follows.

\subsubsection{Non blow-up regions in a HCN molecule}

The schematic figure of this molecule is shown in Fig. 3. This molecule consists of three atoms $\mathrm{H}, \mathrm{C}$ and $\mathrm{N}$. Restricting to the zero total angular momentum, the Hamiltonian can be described by the following three degrees of freedom (dofs), $r$ (distance between $\mathrm{C}$ and $\mathrm{N}$ atom), $R$ (distance between $\mathrm{H}$ and the 


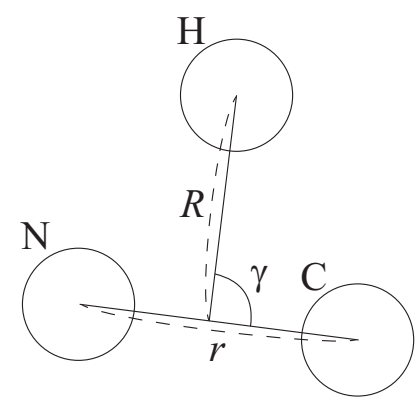

Fig. 3 A schematical figure of HCN molecule

center of mass of $\mathrm{C}$ and $\mathrm{N}$ ) and $\gamma$ (angle between $\mathrm{H}$ and $\mathrm{C}$ as seen from the center of mass of $\mathrm{C}$ and $\mathrm{N}$ ) in the Jacobi coordinate. The corresponding Hamiltonian is

$$
H=\frac{1}{2 \mu} p_{r}^{2}+\frac{1}{2 m} p_{R}^{2}+\frac{1}{2}\left(\frac{1}{\mu r^{2}}+\frac{1}{m R^{2}}\right) p_{\gamma}^{2}+V(r, R, \gamma)
$$

where $\mu=\left(m_{C} m_{N}\right) /\left(m_{C}+m_{N}\right)$ is the reduced mass of the CN diatom, $m=$ $\left(m_{H}\left(m_{C}+m_{N}\right)\right) /\left(\left(m_{H}+m_{C}+m_{N}\right)\right)$ the reduced mass of the full system, and the potential $V(r, R, \gamma)$ is taken from Murrell et al. [79]. This molecule has two minima that have collinear configurations, one is called $\mathrm{HCN}$ and the other is $\mathrm{CNH}$. The potential energy of the saddle located in between the HCN and $\mathrm{CNH}$ wells is $-0.444 \mathrm{kcal} / \mathrm{mol}$. The $\mathrm{HCN}$ and $\mathrm{CNH}$ well and the saddle point that lies between the two wells correspond to $\gamma \approx 0, \approx \pi$ and $\gamma \approx$ \pm 1.168 rad., respectively. In Fig. 4 , we show intersections between non blowup regions $U_{m}(m=4,8,12,16)$ and $p_{r}=p_{\gamma}, p_{R}=0, H=-0.430 \mathrm{kcal} / \mathrm{mol}$ projected on the coordinate space $(r, R, \gamma)$ by using ParaView [80], version 4.10. In Fig. 4, the boundary of the energetically accessible region is plotted in a transparent surface. This surface looks like a bottle and its neck corresponds to the saddle region $\gamma \approx 1.168$ (rad.). This figure indicates that non blow-up regions disappear at the saddle region and, at the perturbation of order 16th, $U_{16}$ cannot cover the whole region inside $\mathrm{HCN}$ basin $\gamma=-1.168 \sim 1.168$ (rad.).

\section{A method of how to improve non blow-up regions}

In this section, we propose a method to improve validity ranges of Lie canonical perturbation theory. In this section and in what follows, we assume $H_{2}(\mathbf{q}, \mathbf{p})$ can be written as $\frac{1}{2} \sum_{i=1}^{n} \omega_{i}\left(q_{i}^{2}+p_{i}^{2}\right)$, which holds if the origin $(\mathbf{q}, \mathbf{p})=\mathbf{0}$ is an elliptic fixed point, where $\omega_{i}\left(\omega_{i}>0\right)$ is a linear frequency of the $i$ th mode. However, it is straightforward to generalize this method to the other types of fixed points. We propose generating functions of a form $\breve{F}_{k}(\mathbf{q}, \mathbf{p})=$ $\left(1-\exp \left(-\frac{\alpha_{k}}{H_{2}^{l}}\right)\right) F_{k}(\mathbf{q}, \mathbf{p})$ where $F_{k}(\mathbf{q}, \mathbf{p})(k=3, \cdots, m)$ are the generating 

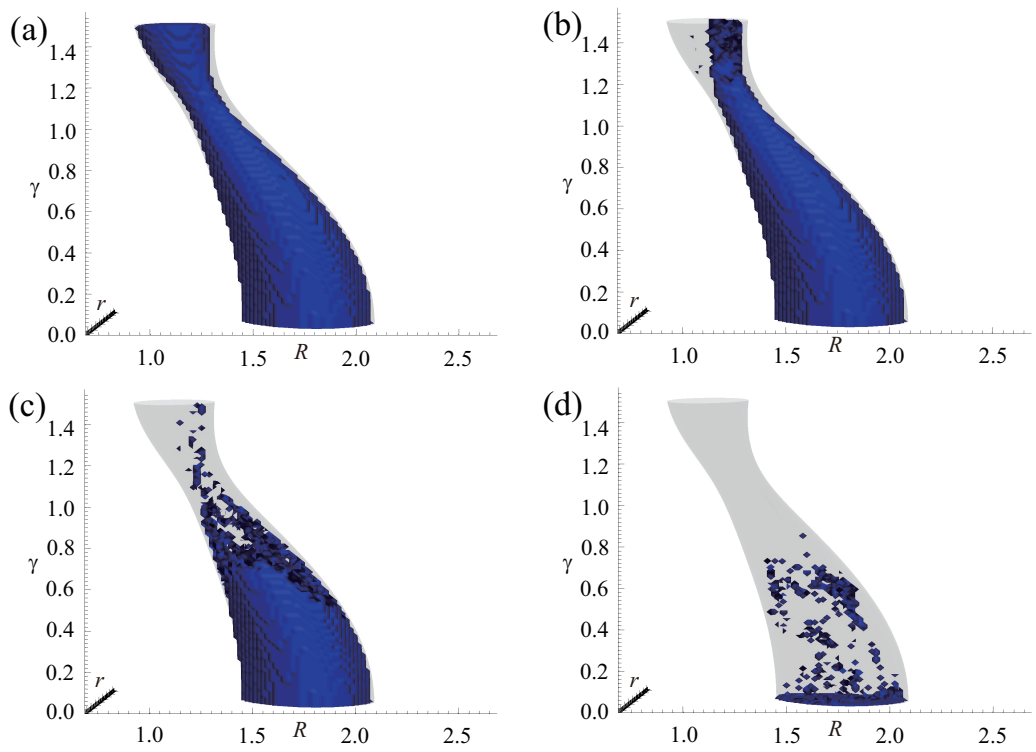

Fig. 4 Non blow-up regions of LCPT by Dragt and Finn: intersections between $U_{m}$ and $p_{r}=p_{\gamma}, p_{R}=0, H=-0.430 \mathrm{kcal} / \mathrm{mol}$ projected on the coordinate space $(r, R, \gamma),((\mathbf{a})$ $m=4$, (b) $m=8$, (c) $m=12$, and (d) $m=16$ ).

functions of LCPT by Dragt and Finn and $l$ and $\alpha_{k}(k=3, \cdots, m)$ are positive real numbers.

First, note that the new generating function $\check{F}_{k}(\mathbf{q}, \mathbf{p})$ has the same Taylor coefficients as $F_{k}(\mathbf{q}, \mathbf{p})$ and thus the resultant Hamiltonian $\check{H}^{(m)}=e^{-\left\{\check{F}_{m}, \cdot\right\}} \ldots e^{-\left\{\check{F}_{3}, \cdot\right\}} H$ has the same normal form as $H^{(m)}=e^{-\left\{F_{m}, \cdot\right\}} \cdots e^{-\left\{F_{3}, \cdot\right\}} H$ up to the order $m$.

Second, due to the factor $\left(1-\exp \left(-\frac{\alpha_{k}}{H_{2}^{l}}\right)\right)$ in front of the generating function, the canonical transformation generated by the new generating function is free from blowing up. To show this, it is sufficient to show that all the solutions of the following differential equations $(3 \leq k \leq m)$,

$$
\begin{aligned}
\frac{d \mathbf{q}}{d \epsilon} & =\frac{\partial \check{F}_{k}(\mathbf{q}, \mathbf{p})}{\partial \mathbf{p}}, \\
\frac{d \mathbf{p}}{d \epsilon} & =-\frac{\partial \check{F}_{k}(\mathbf{q}, \mathbf{p})}{\partial \mathbf{q}},
\end{aligned}
$$

do not blow up for $\epsilon \in[0,1]$. It is because the results of LCPT should be finite if the solutions do not blow up for all $k=3, \cdots, m$. To show this, it is sufficient to show $r_{\omega}=\|(\mathbf{q}, \mathbf{p})\|_{\omega}$ is bounded within the unit time by the time evolution of Eqs. (43) and (44) where $\|\cdot\|_{\omega}$ is a norm induced by a weighted 
inner product

$$
\left\langle\left(\mathbf{q}^{\prime}, \mathbf{p}^{\prime}\right),(\mathbf{q}, \mathbf{p})\right\rangle=\sum_{i=1}^{n} \omega_{i}\left(q_{i}^{\prime} q_{i}+p_{i}^{\prime} p_{i}\right) .
$$

It can be evaluated as

$$
\left|\frac{d \log r_{\omega}}{d \epsilon}\right| \leq C_{k} r_{\omega}^{k-2}\left(1-\exp \left(-\frac{2^{l} \alpha_{k}}{r_{\omega}^{2 l}}\right)\right)
$$

where we set

$$
C_{k}=\max _{\|\mathbf{e}\|_{\omega}=1}\left\|\nabla F_{k}(\mathbf{e})\right\|_{\omega},
$$

which is a finite number. Under the condition $l \geq \frac{k-2}{2}$, the right hand side of Eq. (46) is bounded and thus $r_{\omega}$ has a finite growth rate during the unit time interval. The detailed derivation of Eq. (46) is shown in Sec. 7.2 in Appendix. The condition $l \geq \frac{k-2}{2}$ is a sufficient condition for non blow-up because if $l \geq \frac{k-2}{2}$ holds, the right hand side of Eq. (46) has a finite limit $\lim _{r_{\omega} \rightarrow \infty} 2^{l} \alpha_{k} C_{k} r_{\omega}^{k-2-2 l}$ and, thus, it has a finite maximum value in $[0, \infty]$.

Note that the canonical transformation generated by $\check{F}_{k}$ is no longer analytic. This is due to the fact that the factor $\left(1-\exp \left(-\frac{\alpha_{k}}{H_{2}^{l}}\right)\right)$ is not analytic at the origin $(\mathbf{q}, \mathbf{p})=\mathbf{0}$. In general, the normal form of the Hamiltonian is merely a formal power series and, at best, it is an asymptotic power series with respect to the normalized action-angle variables. This indicates non-existence of analytic canonical transformation that leads to the desired normal form because its existence implies that the original Hamiltonian depends analytically on the normalized action-angle variables. Contrastingly, due to the Borel-Ritt theorem [11], for every formal power series, there exists a $C^{\infty}$ function (which is not necessarily analytic) whose Taylor coefficients are the same as that of the formal power series. Therefore, there may be a canonical transformation of $C^{\infty}$ that leads to the desired normal form. This is one of the reasons why we seek for a non-analytic canonical transformation. A method of how to determine $l$ and $\alpha_{k}(k=3, \cdots, m)$ is shown in Sec. 7.3 in Appendix.

\section{Demonstration of our method to improve the validity range}

In this section, we demonstrate how our method works for the two systems.

4.1 Demonstration of our method in the Hamiltonian system (Eq. (1))

In Fig. 5 (a) and (b), we show that the two actions $I^{(20)}$ and $\check{I}^{(20)}$ along with the true action $I$, where $\check{I}^{(20)}$ is defined as

$$
\check{I}^{(20)}=\frac{1}{2}\left(\left(\check{p}^{(20)}\right)^{2}+\left(\check{q}^{(20)}\right)^{2}\right) .
$$

This figure indicates that the action $\check{I}^{(20)}$ extends smoothly to the outside of 

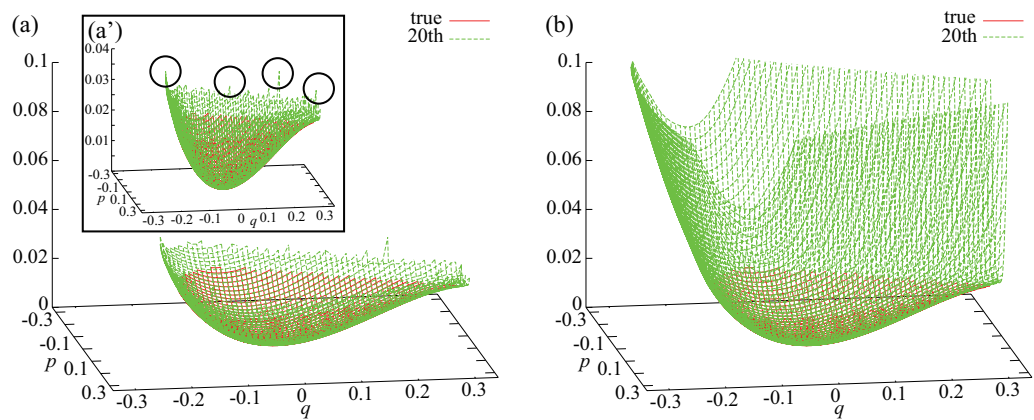

Fig. 5 (a,b) The actions (a) $I^{(20)}$, (b) $\check{I}^{(20)}$ plotted with the true action $I,\left(\mathbf{a}^{\prime}\right)$ a magnified figure of (a) with spurious peaks indicated by the black circles.

the non blow-up region $U_{20}$ whereas $I^{(20)}$ has some spurious peaks indicated by the circles in Fig. $5(\mathrm{a})$. To investigate how the action $\check{I}^{(20)}$ describe the dynamics for the outside region of the separatrix, we superpose the contour surface of $\check{I}^{(20)}$ with the contour lines of the Hamiltonian in Fig. 6 (a). The separatrix is indicated by the pink dotted line in this figure. This figure indicates that the two contour lines are roughly parallel with each other. To quantify it, we plot $\left|\frac{\left\{\check{I}^{(20)}, H\right\}}{\check{I}^{(20)}}\right|$ and $\left|\frac{\left\{I_{\text {trunc }}^{(20)}, H\right\}}{I_{\text {trunc }}^{(20)}}\right|$ in Fig. 6 (b) and (c), respectively. If the contour lines of the actions and the Hamiltonian are parallel with each other, this quantity should be zero. This figure indicates that $\left|\frac{\left\{\check{I}^{(20)}, H\right\}}{\check{I}^{(20)}}\right|$ is smaller than $\left|\frac{\left\{I_{\text {trunc }}^{(20)}, H\right\}}{I_{\text {trunc }}^{(20)}}\right|$ by more than 100 times for the outside of the separatrix whereas $\left|\frac{\left\{\check{I}^{(20)}, H\right\}}{\check{I}^{(20)}}\right|$ is less than 0.1 in the plotted region. Here, we use $I_{\text {trunc }}^{(20)}$ as a reference to compare because the non blow-up region $U_{20}$ is almost the same as the region inside the separatrix (see Fig. 1 (b)) and, thus, it cannot be used to compare with $\check{I}^{(20)}$ outside of the separatrix. Again, note that the true action defined as Eq. (37) does not exist outside of the separatrix but the action $\check{I}^{(20)}$ is well defined even outside of the separatrix and serves as an approximate integral of motion, i.e., $\left|\left\{\check{I}^{(20)}, H\right\}\right| \leq 0.1 \times \check{I}^{(20)}$.

\subsection{Demonstration of our method in the HCN molecule}

In this section, we apply our method to the HCN molecule to demonstrate how our method improves the behavior of the action variables. To demonstrate it, we calculate the actions $I_{i}^{(7)}(i=1,2,3), \check{I}_{i}^{(7)} \quad(i=1,2,3)$ and $I_{\text {trunc, } i}^{(7)} \quad(i=1,2,3)$ along a trajectory at energy $-0.430 \mathrm{kcal} / \mathrm{mol}$, which is beyond the potential energy of the saddle located in between HCN and CNH. Roughly speaking, the 3rd mode $(i=3)$ corresponds to the $\gamma$ direction that leads to structural transi- 

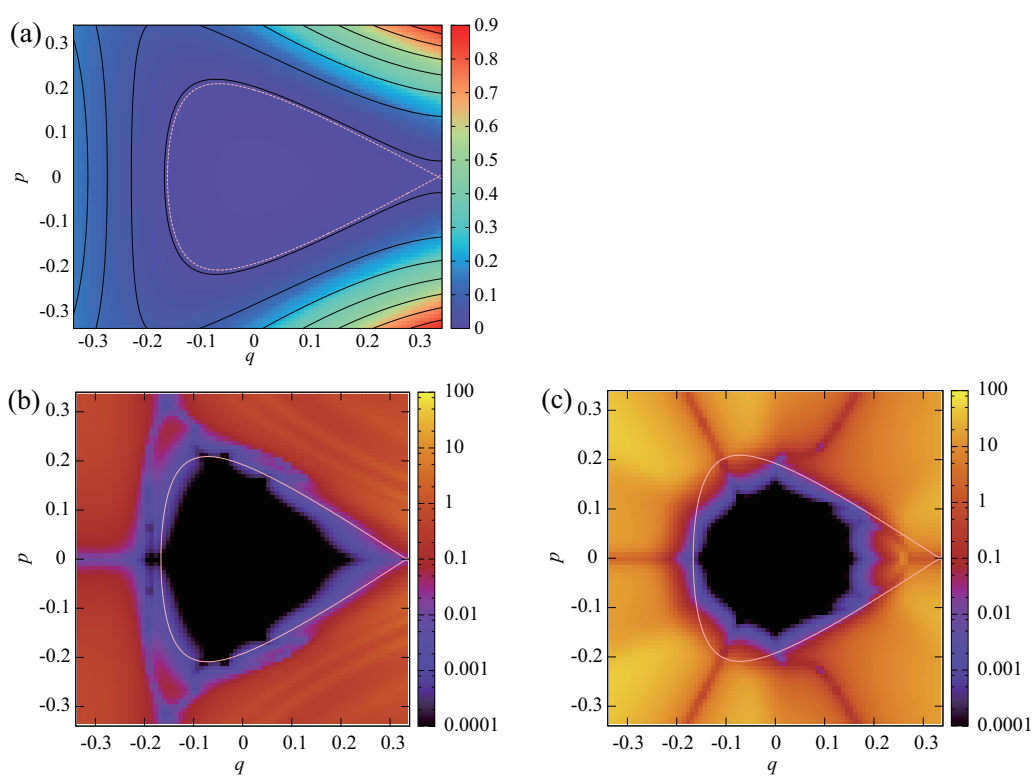

Fig. 6 (a) The contour surface of the action $\check{I}^{(20)}$ plotted with the contour lines of the Hamiltonian in black lines (the separatrix is indicated by the pink dotted curve), (b, c) the Poisson brackets (b) $\left|\left\{\check{I}^{(20)}, H\right\} / \check{I}^{(20)}\right|$ and (c) $\left|\left\{I_{\text {trunc }}^{(20)}, H\right\} / I_{\text {trunc }}^{(20)}\right|$ with the separatrix indicated by the pink dotted curve.

tions between $\mathrm{HCN}$ and $\mathrm{CNH}$ and the other modes $i=1,2$ are the bath modes that weakly couple to the 3rd mode. The perturbation order 7 th is shown to be sufficient to obtain converged actions $[1,2]$ at this energy. In Fig. 7 (a) and (b), we show a typical trajectory of (a) $r, R$ and (b) $\gamma$, respectively. The phase space region $-1.168 \leq \gamma \leq 1.168(\bmod 2 \pi)$ corresponds to the HCN well and this trajectory shows two structural transitions between $\mathrm{HCN}$ and $\mathrm{CNH}$ at the time instances indicated by the arrows, i.e., $t=8.671$ (fs) and $t=6.697 \times 10^{1}$ (fs), in Fig. 7 (b). In the HCN well, we show how the actions evolve in time along the trajectory in Fig. 7 (c) $\left(I_{i}^{(7)}(i=1,2,3), \check{I}_{i}^{(7)}(i=1,2,3)\right)$ and (d) $\left(I_{\text {trunc }, i}^{(7)}(i=1,2,3)\right)$. The actions $I_{\text {trunc, } i}^{(7)}(i=1,2,3)$ change abruptly in time and it is very difficult to extract any insight from the actions but the actions shown in Fig. 7 (c) indicate the existence of the slowly varying actions. However, the actions $I_{i}^{(7)}(i=1,2,3)$ have spurious peaks as indicated by the circles in Fig. 7 (c). These peaks appear when the trajectory comes very close to the edge of the HCN well $(\gamma \approx 1.168$ or $\gamma \approx 2 \pi-1.168)$. Contrastingly, the actions $\check{I}_{i}^{(7)}(i=1,2,3)$ are free from these spurious peaks. Further study is needed to quantify the difference between the two but this demonstration indicates potentiality for our method to suppress these spurious peaks on the edge of non blow-up regions. 

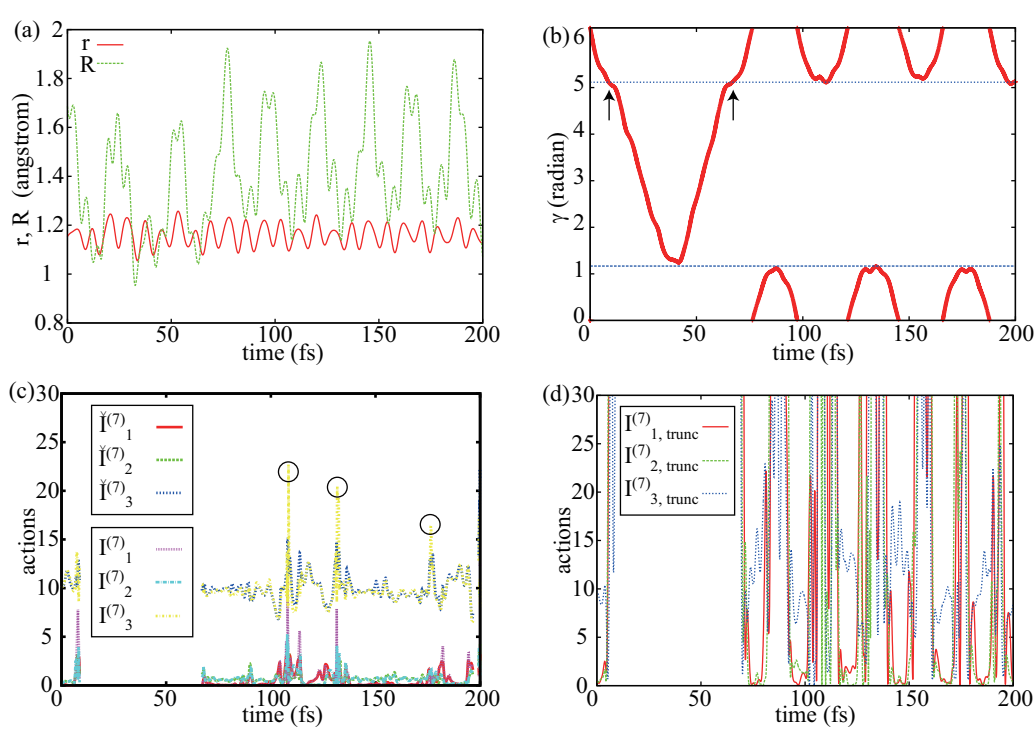

Fig. 7 (a, b) Time series of (a) $r$ and $R$ and (b) $\gamma$ along a trajectory for $2.000 \times 10^{2}$ (fs) with the arrows that indicate time instances when the trajectory exits from the HCN well $(t=8.671(\mathrm{fs}))$ and enters the well $\left(t=6.697 \times 10^{1}(\mathrm{fs})\right),(\mathbf{c})$ the actions $I_{i}^{(7)}(i=1,2,3)$ and $\check{I}_{i}^{(7)}(i=1,2,3)$ with spurious peaks of $I_{3}^{(7)}$ indicated by the circles, (d) the actions $I_{\text {trunc }, i}^{(7)}(i=1,2,3)$.

\section{Conclusions and Discussions}

Validity ranges of Lie Canonical Perturbation Theory (LCPT) have been investigated in terms of non blow-up regions. Non blow-up region of LCPT is a subset of initial conditions in the phase space where the results of the perturbation are finite. Non blow-up region limits the validity ranges of LCPT because the results should be at least finite to validate them. We have investigated how the validity ranges depend on the perturbation order in two systems, one of which is a simple Hamiltonian system with one degree of freedom and the other is a HCN molecule. Our analysis of the former system indicates that non blow-up regions become reduced in size as the perturbation order increases. In case of LCPT by Dragt and Finn and that by Deprit, the non blow-up regions enclose the region inside the separatrix of the Hamiltonian but it may not be the case for LCPT by Hori. We have also analyzed how well the actions constructed by these LCPTs approximate the true action of the Hamiltonian in the non blow-up regions and have found that the conventional truncated LCPT does not work over the whole region inside the separatrix whereas Dragt and Finn's without truncation does. In addition, LCPT by Dragt and Finn leads to smaller errors than those by Deprit. Regarding the width of the non blow-up region and the accuracy inside it, LCPT by Dragt and Finn leads to the best results among the three. Our analysis of the latter system indicates 
that non blow-up regions do not necessarily cover the whole region inside the HCN well.

We have proposed a new perturbation method to improve non blow-up regions and validity ranges inside them. Our method is free from blowing up and retains the same normal form as the conventional LCPT. We demonstrated our method in the two systems and showed that the actions constructed by our method have larger validity ranges than those by the conventional ones and our previous method proposed in $[1,2]$. Previously, Padé approximations have also been used to improve validity ranges of LCPT [81-86]. Empirically, these approximations work well and poles of the Padé approximation tend to clump together in the regions where chaotic motion is observed, such as separatrices or other chaotic regions $[81,84]$. However, even for an entire function that is analytic in the whole complex plane, its Padé approximation can diverge everywhere [87], and thus, it may not be a reliable methd to investigate the phase space geometry. In addition, Teramoto et al. [1,2] demonstrated that Padé approximation does not work for a highly-excited HCN molecule. Contrastingly, our method is free from such a spurious diverging behavior and works even for such highly-excited molecules. Some other possible methods to improve validity ranges are using different styles of normalization [88] and using Kolmogorov normal form $[89,90]$. Both of the methods can be used combining with our method.

Our method can be applied to various subjects in dynamical reaction theory. For example, it would enable us to estimate the time evolution of action variables more precisely than the existing methods, since the action variables constructed by our method are free from blowing up. Thus, it provides us with a new methodology to visualize the Arnold web leading to a better understanding of the dynamical mechanism of intramolecular vibrational-energy redistribution (IVR) [91]. Moreover, the method can be used to investigate how the region around the potential saddle and the well are connected dynamically, since the actions thus constructed offer a better approximation of the real dynamics locally even beyond the separatrix. Therefore, we could evaluate how the stable/unstable manifolds emanating from the normally hyperbolic invariant manifold (NHIM) around the saddle look like in the well even when the energy of the reactive mode is larger than that of the saddle. It would make it possible to understand how the reactive mode obtains energy to go over the saddle from the well and how it loses energy to end up in the well. Results of these studies will be published in near future in separate papers.

\section{Acknowledgments}

We would like to dedicate this article to continuous stimulating and pioneering works by Professor Greg. Ezra in the research field of classical and semiclassical chemical dynamics. HT would like thank Professor Kazuyuki Yagasaki and Zin Arai for their valuable comments on the definition of the action in the Hamiltonian with one degree of freedom and Professor Turgay Uzer for his comment 
on the Padé approximation as an alternative method for improving the validity range. This work has been supported by JSPS, the Cooperative Research Program of "Network Joint Research Center for Materials and Devices", Research Center for Computational Science, Okazaki, Japan, and Grant-in-Aid for challenging Exploratory Research (to TK), and Grant-in-Aid for Scientific Research (B) (to TK) from the Ministry of Education, Culture, Sports, Science and Technology, and Nara Women's University Intramural Grant for Project Research (to MT), Grant-in-Aid for challenging Exploratory Research (to MT), and Grant-in-Aid for Scientific Research (C) (to MT) from the Ministry of Education, Culture, Sports, Science and Technology.

\section{Appendix}

7.1 A blow up method to solve Eqs. (27) and (28)

It is difficult to solve Eqs. (27) and (28) directly because their right hand sides are of order $k-1$ with respect to $\mathbf{q}$ and $\mathbf{p}$ and increase rapidly as $\mathbf{q}$ and $\mathbf{p}$ increase. To solve the differential equation, we introduce the following blow up coordinates [78], $r$ and $\mathbf{e}$ such that $(\mathbf{q}, \mathbf{p})=r \mathbf{e}$ and $\mathbf{e} \cdot \mathbf{e}=1$. In addition to them, we introduce a scaled virtual time $s$ so that $\epsilon$ increases slower as the solution approaches to the infinity such that $d \epsilon=\frac{1}{r^{(k-2)}} d s$. In terms of the blow up coordinates and the scaled virtual time, Eqs. (27) and (28) can be written as

$$
\begin{aligned}
\frac{d \log r}{d s} & =\Theta_{k}(\mathbf{e}) \\
\frac{d \mathbf{e}}{d s} & =\left(\left.\frac{\partial F_{k}(\mathbf{q}, \mathbf{p})}{\partial \mathbf{p}}\right|_{(\mathbf{q}, \mathbf{p})=\mathbf{e}},-\left.\frac{\partial F_{k}(\mathbf{q}, \mathbf{p})}{\partial \mathbf{q}}\right|_{(\mathbf{q}, \mathbf{p})=\mathbf{e}}\right)-\Theta_{k}(\mathbf{e}) \mathbf{e} \\
\frac{d \epsilon}{d s} & =\frac{1}{r^{(k-2)}}
\end{aligned}
$$

where $\Theta_{k}(\mathbf{e})$ is defined as

$$
\Theta_{k}(\mathbf{e})=\mathbf{e} \cdot\left(\left.\frac{\partial F_{k}(\mathbf{q}, \mathbf{p})}{\partial \mathbf{p}}\right|_{(\mathbf{q}, \mathbf{p})=\mathbf{e}},-\left.\frac{\partial F_{k}(\mathbf{q}, \mathbf{p})}{\partial \mathbf{q}}\right|_{(\mathbf{q}, \mathbf{p})=\mathbf{e}}\right) .
$$

Eqs. (49), (50) and (51) can be solved stably because norms of their right hand sides are bounded by a finite value, i.e., $\max _{\mathbf{e} \cdot \mathbf{e}=1}\left\|\nabla F_{k}\right\|$, where $\|\cdot\|$ is Euclidean norm. In this paper, we integrate this differential equation by using Stepper Dropper853 [92], which is 8th order Runge-Kutta method with step size control under the constraint $\mathbf{e} \cdot \mathbf{e}=1$ until $\epsilon \leq 1$. We use the double precision to integrate them and if the value of $\log r$ exceeds the logarithm of the maximum value of double defined in the standard C++ library [93],

std: :numeric_limits<double> : : infty () 
that is $1.79769 \mathrm{e}+308$ in our current environment, we regard the solution as one that blows up.

This method can be also used to solve differential equations induced by generating functions in LCPTs by Hori (Eqs. (32) and (33)) and Deprit (Eqs. (35) and (36)). However, those generating functions are not homogeneous polynomials and thus this method needs to be adopted for them. To solve them accurately, we decompose the phase space into two regions: one is a region where the highest order terms in the generating function dominate and the other is its complement. In the former region, by introducing the blow up coordinate and the scaled virtual time, the differential equations can be written as Eqs. (49), (50) and (51) plus some correction terms of order $1 / r$. They can be integrated in the same manner as above. In the latter region, since the lower order terms still dominate, we can directly integrate the differential equations.

\subsection{A derivation of Eq. (46)}

The $\epsilon$-derivative of $r_{\omega}$ can be calculated as follows,

$$
\begin{aligned}
\frac{d r_{\omega}}{d \epsilon} & =\frac{1}{r_{\omega}} \sum_{i=1}^{n} \omega_{i}\left(q_{i} \frac{d q_{i}}{d \epsilon}+p_{i} \frac{d p_{i}}{d \epsilon}\right), \\
& =\frac{1}{r_{\omega}} \sum_{i=1}^{n} \omega_{i}\left(q_{i} \frac{\partial \check{F}_{k}}{\partial p_{i}}-p_{i} \frac{\partial \check{F}_{k}}{\partial q_{i}}\right), \\
& =\frac{1}{r_{\omega}} \sum_{i=1}^{n} \omega_{i}\left(q_{i} \frac{\partial F_{k}}{\partial p_{i}}-p_{i} \frac{\partial F_{k}}{\partial q_{i}}\right)\left(1-\exp \left(-\frac{2^{l} \alpha_{k}}{r_{\omega}^{2 l}}\right)\right) .
\end{aligned}
$$

Define $\mathbf{e}$ as $(\mathbf{q}, \mathbf{p})=r_{\omega} \mathbf{e}$, then, $\|\mathbf{e}\|_{\omega}=1$ holds. By using this, we get

$$
\begin{aligned}
\frac{d r_{\omega}}{d \epsilon} & =\sum_{i=1}^{n} \omega_{i}\left(e_{i} \frac{\partial F_{k}}{\partial p_{i}}-e_{i+n} \frac{\partial F_{k}}{\partial q_{i}}\right)\left(1-\exp \left(-\frac{2^{l} \alpha_{k}}{r_{\omega}^{2 l}}\right)\right), \\
& =r_{\omega}^{k-1} \sum_{i=1}^{n} \omega_{i}\left(\left.e_{i} \frac{\partial F_{k}}{\partial p_{i}}\right|_{(\mathbf{q}, \mathbf{p})=\mathbf{e}}-\left.e_{i+n} \frac{\partial F_{k}}{\partial q_{i}}\right|_{(\mathbf{q}, \mathbf{p})=\mathbf{e}}\right)\left(1-\exp \left(-\frac{2^{l} \alpha_{k}}{r_{\omega}^{2 l}}\right)\right) .
\end{aligned}
$$

Finally, we get

$$
\left|\frac{d \log r_{\omega}}{d \epsilon}\right| \leq r_{\omega}^{k-2}\left(1-\exp \left(-\frac{2^{l} \alpha_{k}}{r_{\omega}^{2 l}}\right)\right) \max _{\|\mathbf{e}\|_{\omega}=1}\|\nabla F(\mathbf{e})\|_{\omega} .
$$

by using the following inequality,

$$
\begin{aligned}
& -\max _{\|\mathbf{e}\|_{\omega}=1}\|\nabla F(\mathbf{e})\|_{\omega} \\
& \qquad \sum_{i=1}^{n} \omega_{i}\left(\left.e_{i} \frac{\partial F_{k}}{\partial p_{i}}\right|_{(\mathbf{q}, \mathbf{p})=\mathbf{e}}-\left.e_{i+n} \frac{\partial F_{k}}{\partial q_{i}}\right|_{(\mathbf{q}, \mathbf{p})=\mathbf{e}}\right) \\
& \leq \max _{\|\mathbf{e}\|_{\omega}=1}\|\nabla F(\mathbf{e})\|_{\omega} .
\end{aligned}
$$


7.3 A method to determine $l$ and $\alpha_{k}(k=3, \cdots, m)$ in Sec. 3

As pointed out in Sec. 3, the condition $l \geq \frac{k-2}{2}$ is a sufficient condition for the right hand side of Eq. (46) is bounded. Here, we choose $l=k-2$ for simplicity but this choice may not be the best choice. Further study is needed to find an optimal power $l$. Under this choice, the maximum value of the right hand side of Eq. (46) can be evaluated as $2^{\frac{k-2}{2}} C_{k} \alpha_{k}^{\frac{1}{2}} f^{*}$ and this maximum is attained at $r_{\omega}=\sqrt{2 \alpha_{k}^{\frac{1}{k-2}}} r^{*}$, where $f^{*}\left(\approx 6.38173 \times 10^{-1}\right)$ and $r^{*}\left(\approx 8.92135 \times 10^{-1}\right)$ the maximum and the argument that attains the maximum of the function $f(r)=r\left(1-\exp \left(\frac{1}{r^{2}}\right)\right)(r \geq 0)$, respectively.

For the right hand side of Eq. (46) to be of order 1, i.e., $2^{\frac{k-2}{2}} C_{k} \alpha_{k}^{\frac{1}{2}} f^{*} \sim 1$, $\alpha_{k}$ should satisfy $\alpha_{k} \sim \frac{1}{2^{k-2}}\left(\frac{1}{f^{*} C_{k}}\right)^{2}$. This is how we determine $\alpha_{k}(k=3, \cdots, m)$. If the $\alpha_{k}$ is chosen as this,

$$
\left|\frac{r_{\omega}^{(k+1)}-r_{\omega}^{(k)}}{r_{\omega}^{(k)}}\right| \lesssim 1
$$

holds for $k=3, \cdots, m-1$. This should hold if $(k+1)$ th order perturbation acts as a correction to the result up to the $k$ th order perturbation.

Note that, if $\alpha_{k}$ becomes smaller, the deviation between $F_{k}$ and $\check{F}_{k}$ becomes larger. Therefore, it is favorable if $\alpha_{k}$ is chosen as large as possible. Since $\alpha_{k}$ is inversely proportional to $C_{k}^{2}$, it may also be important to suppress the growth of $F_{k}$ without normalizing near-resonant terms in LCPT.

\section{References}

1. H. Teramoto and T. Komatsuzaki. Exploring remnant of invariants buried in a deep potential well in chemical reactions. J. Chem. Phys., 129:094302, 2008.

2. H. Teramoto and T. Komatsuzaki. Probing remnants of invariant s to mediate energy exchange in highly-chaotic many-dimensional systems. Phys. Rev. E, 78:017202, 2008.

3. A. J. Lichtenberg and M. A. Lieberman. Regular and Chaotic Dynamics. Springer, New York, second edition edition, 1991.

4. G. Hori. Theory of general perturbations with unspecified canonical variables. Pub. Astro. Soc. Japan, 18:287, 1966.

5. G. Hori. Non-linear coupling of two harmonic oscillations. Pub. Astro. Soc. Japan, 19:229, 1967.

6. A. Deprit. Canonical transformations depending on a small parameter. Celest. Mech., 1:12, 1969.

7. A. J. Dragt and J. M. Finn. Lie series and invariant functions for analytic symplectic maps. J. Math. Phys., 17:2215, 1976.

8. J. A. Campbell and W. H. Jefferys. Equivalence of the perturbation theories of Hori and Deprit. Celes. Mech., 2:467, 1970.

9. J. R. Cary. Lie transform perturbation theory for Hamiltonian systems. Phys. Rev., 79:129, 1981.

10. P.V. Koseleff. Comparison between Deprit and Dragt-Finn perturbation methods. Celes. Mech. Dyn. Astron., 58:17, 1994.

11. J. Murdock. Normal Forms and Unfoldings for Local Dynamical Systems. Springer Monographs in Mathematics. Springer, New York, first edition, 2003. 
12. C. L. Siegel. On the Integrals of Canonical Systems. Ann. Math., 42:806, 1941.

13. A. D. Bryuno. Normal form of real differential equations. Math. Note, 18:722, 1975.

14. A. D. Bryuno. Divergence of a real normalizing transformation. Math. Note, 31:207, 1982.

15. H. Ito. Convergence of Birkhoff normal forms for integrable systems. Comment. Math. Helvetici, 64:412, 1989.

16. H. Ito. Integrability of Hamiltonian systems and Birkhoff normal forms in the simple resonance case. Math. Ann., 292:411, 1992.

17. A. D. Bruno and S. Walcher. Symmetries and Convergence of Normalizing Transformations. J. Math. Anal. Appl., 183:571, 1994.

18. G. Cicogna. On the Convergence of Normalizing Transformations in the Presence of Symmetries. J. Math. Anal. Appl., 199:243, 1996.

19. T. Kappeler, Y. Kodama, and A. Némethi. On the Birkhoff Normal Form of a Completely Integrable Hamiltonian System Near a Fixed Point with Resonance. Ann. Scuola Norm. Sup. Pisa Cl. Sci., XXVI:623, 1998.

20. S. Walcher. On Convergent Normal Form Transformations in Presence of Symmetries. J. Math. Anal. Appl., 244:17, 2000.

21. P. Pérez-Marco. Total Convergence or General Divergence in Small Divisors. Commun. Math. Phys., 223:451, 2001.

22. G. Cicogna and S. Walcher. Convergence of Normal Form Transformations: The Role of Symmetries. Acta Appl. Math., 70:95, 2002.

23. N. T. Zung. Convergence versus Integrability in Birkhoff Normal Form. Ann. Math., 161:141, 2005

24. H. Chiba. Extension and Unification of Singular Perturbation Methods for ODEs Based on the Renormalization Group Method. SIAM j. on Appl. Dyn. Syst., 8:1066, 2009.

25. L. Markus and K. R. Meyer. Generic hamiltonian dynamical systems are neither integrable nor ergodic. Mem. Amer. Math. Soc., 144, 1974.

26. W.S. Koon, M.W. Lo, J.E. Marsden, and S.D. Ross. Heteroclinic connections between periodic orbits and resonance transitions in celestial mechanics. Chaos, 10:427, 2000

27. C. Jaffe, S. D. Ross, M. W. Lo, J. Marsden, D. Farrelly, and T. Uzer. Statistical theory of asteroid escape rates. Phys. Rev. Lett., 89:011101, 2002.

28. J. von Milczewski, G. H. F. Diercksen, and T. Uzer. Computation of the Arnol'd Web for the Hydrogen Atom in Crossed Electric and Magnetic Fields. Phys. Rev. Lett., $76: 2890,1996$.

29. T. Uzer, C. Jaffé, J. Palacián, P. Yanguas, and S. Wiggins. The geometry of reaction dynamics. Nonlinearity, 15:957, 2002.

30. T. Komatsuzaki and R. S. Berry. Regularity in chaotic reaction paths. I. Ar 6 . J. Chem. Phys., 110:9160-9173, 1999.

31. T. Komatsuzaki and R. S. Berry. Regularity in chaotic reaction path II: $\operatorname{Ar}_{6}$-energy dependence and visualization of the reaction bottleneck-. Phys. Chem. Chem. Phys. 1:1387, 1999.

32. T. Komatsuzaki and R. S. Berry. Local regularity and non-recrossing path in transition states -a new strategy in chemical reaction theories. J. Mol. Struct. (Theochem), 506:55, 2000.

33. T. Komatsuzaki and R. S. Berry. Regularity in chaotic reaction paths. III: -local invariances at the reaction bottleneck-. J. Chem. Phys., 115:4105, 2001.

34. T. Komatsuzaki and R. S. Berry. Dynamical hierarchy in transition states: Why and how does a system climb over the mountain? Proc. Nat. Acad. Sci. USA, 98:7666, 2001.

35. T. Komatsuzaki and R. S. Berry. A dynamical propensity rule of transitions in chemical reactions. J. Phys. Chem. A, 106:10945, 2002.

36. T. Komatsuzaki and R. S. Berry. Chemical reaction dynamics: Many-body chaos and regularity. Adv. Chem. Phys., 123:79, 2002.

37. T. Komatsuzaki and M. Nagaoka. Study on "regularity" of the barrier recrossing motion. J. Chem. Phys., 105:10838, 1996.

38. T. Komatsuzaki and M. Nagaoka. A dividing surface free from a barrier recrossing motion in many-body systems. Chem. Phys. Lett., 265:91, 1997.

39. S. Kawai, Y. Fujimura, O. Kajimoto, T. Yamashita, C.-B. Li, T. Komatsuzaki, and M. Toda. Dimension reduction for extracting geometrical structure of multidimensional phase space: Application to fast energy exchange in the reaction $\mathrm{O}\left({ }^{1} \mathrm{D}\right)+\mathrm{N}_{2} \mathrm{O} \rightarrow \mathrm{NO}+\mathrm{NO}$. Physical Review A, 75:022714, 2007. 
40. S. Kawai and T. Komatsuzaki. Robust existence of a reaction boundary to separate the fate of a chemical reaction. Phys. Rev. Lett., 105:048304, 2010.

41. C.-B. Li, Y. Matsunaga, M. Toda, and T. Komatsuzaki. Phase space reaction network on a multisaddle energy landscape: Hcn isomerization. J. Chem. Phys., 123:184301, 2005.

42. H. Waalkens, A. Burbanks, and S. Wiggins. Phase space conduits for reaction in multidimensional systems, HCN isomerization in three dimensions. J. Chem. Phys., 121:6207, 2004 .

43. T. Bartsch, R. Hernandez, and T. Uzer. Transition state in a noisy environment. Phys. Rev. Lett., 95:058301, 2005.

44. T. Bartsch, T. Uzer, and R. Hernandez. Stochastic transition states: Reaction geometry amidst noise. J. Chem. Phys., 123:204102, 2005.

45. T. Bartsch, T. Uzer, J. M. Moix, and R. Hernandez. Identifying reactive trajectories using a moving transition state. J. Chem. Phys., 124:244310, 2006.

46. S. Kawai and T. Komatsuzaki. Dynamical reaction coordinate buried in thermal fluctuation i: time-dependent normal form theory for multidimensional underdamped langevin equation. J. Chem. Phys., 131:224505, 2009.

47. S. Kawai and T. Komatsuzaki. Dynamical reaction coordinate buried in thermal fluctuation ii: numerical examples. J. Chem. Phys., 131:224506, 2009.

48. S. Kawai and T. Komatsuzaki. Hierarchy of reaction dynamics in a thermally fluctuating environment. Phys. Chem. Chem. Phys., 12:7626-7635, 2010.

49. S. Kawai and T. Komatsuzaki. Nonlinear dynamical effects on reaction rate constants in thermally fluctuating environments. Phys. Chem. Chem. Phys., 12:7636-7647, 2010.

50. S. Kawai and T. Komatsuzaki. Dynamical reaction coordinate in thermally fluctuating environment in the framework of multidimensional generalized langevin equations. Phys. Chem. Chem. Phys., 12:15382-15391, 2010.

51. L. E. Fried and G. S. Ezra. Semiclassical quantization using perturbation theory: algebraic quantization of multidimensional systems. J. Chem. Phys., 86:6270, 1987.

52. L. E. Fried and G. S. Ezra. Perturb: a special-purpose algebraic manipulation program for classical perturbation theory. Comp. Phys. Comm., 51:103, 1988.

53. L. E. Fried and G. S. Ezra. Semiclassical quantization of polyatomic molecules: some recent developments. J. Phys. Chem., 92:3144, 1988.

54. S. Kawai and T. Komatsuzaki. Quantum reaction boundary to mediate reactions in laser fields. J. Chem. Phys., 134:024317, 2011.

55. S. Kawai and T. Komatsuzaki. Laser control of chemical reactions by phase space structures. Bull. Chem. Soc. Japan, 85:854-861, 2012.

56. A. Giorgilli and L. Galgani. Rigorous estimates for the series expansions of hamiltonian perturbation theory. Celes. Mech., 37:95, 1985.

57. V. Arnold. Instabilities in dynamical systems with several degrees of freedom. Sov. Math. Dokl., 5:581, 1964.

58. B. V. Chirikov. A universal instability of many-dimensional oscillator systems. Phys. Rep., 52:263, 1979.

59. M. Guzzo, E. Lega, and C. Froeschlé. A numerical study of the topology of normally hyperbolic invariant manifolds supporting arnold diffusion in quasi-integrable systems. Physica D, 238:1797, 2009.

60. P. M. Cincottaa, C. Efthymiopoulosb, C. M. Giordanoa, and M. F. Mestrea. Chirikov and nekhoroshev diffusion estimates: Bridging the two sides of the river. Physica D, 266:49, 2014.

61. C.C. Martens, M.J. Davis, and G.S. Ezra. Local frequency analysis of chaotic motion in multidimensional systems: energy transport and bottlenecks in planar OCS. Chem. Phys. Lett., 142:519, 1987.

62. K.M. Atkins and D.E. Logan. Intersecting resonances as a route to chaos: classical and quantum studies of a three-oscillator model. Phys. Lett. A, 162:255, 1992.

63. C. Froeschlé, M. Guzzo, and E. Lega. Graphical evolution of the arnold web: from order to chaos. Science, 289:2108, 2000.

64. C. Chandre, S. Wiggins, and T. Uzer. Time-frequency analysis of chaotic systems. Physica D, 181:171, 2003. 
65. A.Shojiguchi, C-B. Li, T.Komastuzaki, and M.Toda. Wavelet Analysis and Arnold Web Picture for Detecting Energy Transfer in Hamiltonian Dynamical System. Laser Physics, 17:1097, 2006.

66. J. Laskar. Frequency analysis for multi-dimensional systems. Global dynamics and diffusion. Physica D, 67:257, 1993.

67. S. Honjo and K. Kaneko. Structure of Resonances and Transport in Multidimensional Hamiltonian Dynamical Systems. Adv. Chem. Phys., 130B:437, 2003.

68. A. Semparithi and S. Keshavamurthy. Intramolecular vibrational energy redistribution as state space diffusion: Classical-quantum correspondence. J. Chem. Phys., 125:141101, 2006.

69. A.Shojiguchi, C-B. Li, T.Komastuzaki, and M.Toda. Fractional behavior in nonergodic reaction processes of isomerization. Phys. Rev, E75:035204(R), 2007.

70. A.Shojiguchi, C-B. Li, T.Komastuzaki, and M.Toda. Fractional behavior in multidimensional Hamiltonian systems describing reactions. Phys. Rev, E76:056205, 2007.

71. S. Wiggins. On the geometry of transport in phase space I. Transport in $k$-degree-offreedom Hamiltonian Systems, $2 \leq k \leq \infty$. Physica D, 44:471, 1990.

72. R. E. Gillilan and G. S. Ezra. Transport and turnstiles in multidimensional hamiltonian mappings for unimolecular fragmentation: Application to van der Waals predissociation. J. Chem. Phys., 94:2648, 1991.

73. M. Toda. Crisis in chaotic scattering of a highly excited van der waals complex. Phys. Rev. Lett., 74:2670, 1995.

74. A.Shojiguchi, C-B. Li, T.Komastuzaki, and M.Toda. Dynamical Foundation and Limitation of Statistical Reaction Theory. Communications in Nonlinear Science and Numerical Simulation, 13:857, 2008.

75. H. Goldstein, C. P. Poole Jr., and J. L. Safko. Classical Mechanics. Addison-Wesley, third edition, 2001

76. E. E. Coddington. Theory of Ordinary Differential Equations. Krieger Pub Co, 1984.

77. A. J. Dragt and J. M. Finn. Normal form form mirror machine hamiltonians. J. Math. Phys., 20:2649, 1979.

78. C. S. Coleman. Boundedness and unboundedness in polynomial differential systems. Nonlin. Analy., Theor. Meth. Appl., 8:1287, 1984.

79. J. N. Murrell, S. Carter, and L. O. Halonen. Frequency optimized potential energy functions for the ground-state surfaces of hcn and hcp. J. Mol. Spectrosc., 93:307, 1982.

80. J. Ahrens, B. Geveci, and C. Law. ParaView: An End-User Tool for Large Data Visualization. In C. Hansen and C. Johnson, editors, The Visualization Handbook, page 717. Academic Press, 2005.

81. R. B. Shirts and W. P. Reinhardt. Approximate constants of motion for classically chaotic vibrational dynamics: Vague tori, semiclassical quantization, and classical intramolecular energy flow. J. Chem. Phys., 77:5204, 1982.

82. M. K. Ali, W. R. Wood, and J. S. Devitt. On the summation of the Birkhoff-Gustavson normal form of an anharmonic oscillator. J. Math. Phys., 27:1806, 1986.

83. M. K. Ali and W. R. Wood. The Birkhoff-Gustavson Normal Form of Double-Well Anharmonic Oscillators. Prog. Theor. Phys., 78:766, 1987.

84. M. Robnik. On the Padé approximations to the Birkhoff-Gustavson normal form. $J$. Phys. A: Math Gen., 26:7427, 1993.

85. C. B. Li, A. Shojiguchi, M. Toda, and T. Komatsuzaki. Definability of no-return transition states in high energy regime above threshold. Phys. Rev. Lett., 97:028302, 2006.

86. H. Teramoto and K. Takatsuka. Local integrals and their globally connected invariant structure in phase space giving rise to a promoting mode of chemical reaction. J. Chem. Phys., 126:124110, 2007.

87. G. A. Baker Jr. and P. Graves-Morris. Padé Approximants. Cambridge University Press, second edition, 1996.

88. M. Kaluža and M. Robnik. Improved accuracy of the Birkhoff-Gustavson normal form and its convergence properties. J. Phys. A: Math Gen., 25:5311, 1992.

89. V. I. Arnold. Proof of a theorem of A. N. Kolmogorov on the invariance of quasi-periodic motions under small perturbations of the Hamiltonian. Russ. Math. Surv., 18:9, 1963.

90. F. Gabern, À. Jorba, and U. Locatelli. On the construction of the Kolmogorov normal form for the Trojan asteroids. Nonlinearity, 18:1705, 2005. 
91. T. Uzer. Theories of intramolecular vibrational energy transfer. Phys. Rep., 199:73, 1991.

92. W. H. Press, S. A. Teukolosky, W. T. Vetterling, and B. P. Flannery. Numerical Recipes, The Art of Scientific Computing. International Series of Monographs on Chemistry. Cambridge University Press, 3rd edition edition, 2007.

93. B. Strpistrup. Programming: Principles and Practice Using $C++$. Addison-Wesley Professional, second edition edition, 2008. 